\title{
THE INJURED
}

They are so misshapen so badly crippled not even surgery can alter nor dark cosmetics mask them. The pain at which they wince as familiarly as at the glare of morning has traced remoteness on their faces.

Thus if they appear to descend infrequently from the high plateaus they have withdrawn to this should not be attributed to fear but constant fatigue the thinness of that air the labored breathing to which they do not adapt. 